\title{
Formas lipídicas de anfotericina
}

\author{
Martha C. Botero, Marcela Puentes-Herrera y Jorge A. Cortés
}

\begin{abstract}
Universidad Nacional de Colombia, Bogotá, Colombia. Facultad de Medicina Departamento de Medicina Interna (MCB, MPH).

Grupo de Investigación en Enfermedades Infecciosas (JAC)

No hay conflictos de interés que declarar. Este estudio no tuvo financiación directa.

Recibido: 29 de agosto de 2013 Aceptado: 2 de julio de 2014

Correspondencia a: Jorge A. Cortés Luna jacortesı@unal.edu.co
\end{abstract}

\section{Introducción}

$\mathrm{E}$ $\mathrm{n}$ los últimos años ha aumentado el número de personas con algún grado de inmunosupresión, como pacientes con infección por VIH/SIDA, receptores de trasplantes de órganos, enfermedades autoinmunes y usuarios de la unidad de cuidado intensivo (UCI). Esto, sumado al progreso de la medicina con quimioterapias más agresivas, el uso de corticosteroides y otros inmunosupresores de forma más frecuente, y el desarrollo y uso de antimicrobianos de amplio espectro, ha llevado a una mayor incidencia de enfermedades fúngicas sistémicas ${ }^{1}$.

Los patógenos más frecuentemente aislados incluyen Candida spp, Aspergillus spp, y zigomicetos. En los Estados Unidos de América (E.U.A.), la mayor prevalencia corresponde a Candida spp. y Aspergillus spp., con 42 y $29 \%$, respectivamente. El $29 \%$ restante es encabezado por zigomicetos y especies de Fusarium. La incidencia de candidiasis invasora en esta población es de 72 a 228 por 1.000 .000 de personas, con una mortalidad de $10 \mathrm{a} 49 \%{ }^{1}$. En América Latina se documentó una alta frecuencia en los hospitales, con una incidencia de 1,18 por 1.000 admisiones en un estudio multicéntrico latinoamericano ${ }^{2}$. Estudios en Brasil sugieren una frecuencia de candidemia en $32 \%$ de pacientes con malignidad hematológica y $68 \%$ de pacientes con tumores sólidos ${ }^{3}$. En un estudio llevado a cabo en un hospital universitario en Chile, se evaluaron pacientes en UCI y hubo una frecuencia de candidemia de $33,9 \%$, candidiasis invasora diseminada de $41,5 \%$ y de candidiasis invasora local de $24,5 \%{ }^{4}$. Entretanto, la micosis invasora por Aspergillus spp. presenta una incidencia de 12 a 34 por 1.000 .000 de habitantes con una mortalidad superior a $85 \%{ }^{1}$.

\section{Anfotericina B}

Existen tres grupos farmacológicos para el manejo de las infecciones fúngicas sistémicas: los polienos, los azoles y las equinocandinas. En el primer grupo encontramos a anfotericina $\mathrm{B}(\mathrm{AmB})^{1}$, considerada el estándar de oro como tratamiento antifúngico con base en su amplio espectro ${ }^{1,5,6}$, aunque su uso ha cambiado por la aparición de medicamentos igualmente efectivos y más seguros en diversos escenarios clínicos (candidiasis, aspergilosis, etc.). Su espectro microbiológico se anota en la Tabla $1^{1,5,6}$.

En esencia, AmB es un producto natural originado de Streptomyces nodosus, que hace parte del grupo de macrólidos, caracterizado por un anillo macrocíclico de lactona. Posee un dominio hidrofóbico y uno hidrofílico lo cual le da su característica anfipática que le confiere baja solubilidad en soluciones acuosas a pH fisiológico. Esto determina que requiera la formación de un complejo con otro agente para su uso clínico, el desoxicolato sódico?

Debido a su baja solubilidad en agua, la biodisponibilidad oral también es baja. Por esto se prefiere el uso de vías adicionales para la administración. Anfotericina B puede ser administrada por vía intravenosa, intratecal, intra-articular, intravesical, dentro de lesiones e infundido en sitios quirúrgicos ${ }^{8}$.

\section{Mecanismo de acción}

La molécula hidrofóbica de AmB se une a la molécula de ergosterol de la pared celular fúngica y juntas producen agregados que forman poros trans-membranas. Esto causa despolarización de la membrana con posterior incremento 
de la permeabilidad de membrana a protones y cationes monovalentes, y permite el paso de moléculas intracelulares al medio externo, ocasionando un desbalance osmótico y finalmente la muerte celular?.

Anfotericina B tiene también la capacidad de unirse a colesterol, aunque con menor afinidad, lo que se cree es responsable del mayor potencial tóxico. Sin embargo, es importante aclarar que la mayor afinidad por el ergosterol es lo que define la selectividad por agentes fúngicos ${ }^{7}$.

Debido a la alta frecuencia de toxicidad por el uso de AmB desoxicolato, se ha intentado disminuir su efecto sobre las células del hospedero, al combinarla en formas lipídicas.

\section{Presentaciones lipídicas de anfotericina B}

Existen cuatro formulaciones lipídicas. Si bien también son llamadas liposomales, realmente sólo dos de éstas lo son. La Tabla 2 muestra las diferencias entre las distintas presentaciones $^{7,10-16}$.

\section{Anfotericina B liposomal}

$\mathrm{Su}$ presentación es en polvo para reconstitución, se encuentra en el mercado bajo el nombre de AmBisome ${ }^{\circledR}$ (Gilead Sciences) y en Chile se consigue otra presentación de origen en India (Cipla). Son esferas de 50-100 $\mu \mathrm{m}$ de diámetro y están compuestas por fosfatidilcolina hidrogenada de soya, colesterol (25\%), unidos estéricamente a diesteril fosfatidilglicerol (DSPG por sus siglas en inglés) y $\mathrm{AmB}$ (en una relación nueve moléculas de lípidos y una de anfotericina, 5\%). De manera similar a la AmB desoxicolato, crea un poro a través de la membrana fúngica; sin embargo, cada molécula de AmB dentro del liposoma

\section{Tabla 1. Espectro antimicótico de anfotericina B}

\begin{tabular}{lll} 
Adecuada susceptibilidad & Susceptibilidad disminuida & Resistencia \\
Candida albicans & Candida krusei & Candida lusitaniae \\
Candida glabrata & Aspergillus flavus & Aspergillus terreus \\
Candida parapsilosis & Fusarium spp. & Scedosporium prolificans \\
Candida tropicalis & Scedosporium apiospermum & \\
Cryptococus neoformans & Absidia corymbifera \\
Aspergillus fumigatus & Apophysomyces elegans \\
Aspergillus niger & Basidiobolus ranarum \\
Coccidiodes spp. & Conidiobolus coronatus \\
Blastomyces spp. & Conidiobolus incongruus \\
Histoplasma spp. & Cunninnghamella bertholletiae \\
& Cokeromyces recurvatus \\
& Mucor indicus \\
& Rhizomucor pusillus \\
& Rhizopus pryzae \\
& Saksenada vasiformis \\
& Syncephalastrum racemosum \\
\hline \multirow{2}{*}{ Información compilada de referencias 1, 5 y 6.} & \\
\hline
\end{tabular}

Tabla 2. Diferencias entre las diferentes presentaciones de anfotericina B (AmB)

\begin{tabular}{|c|c|c|c|c|c|}
\hline & \multirow[t]{2}{*}{ AmB desoxicolato } & \multicolumn{4}{|c|}{ AmB lipídicas } \\
\hline & & AmB liposomal & $\begin{array}{l}\text { AmB liposomal } \\
\text { (India) }\end{array}$ & $\begin{array}{c}\text { AmB } \\
\text { Dispersión coloidal }\end{array}$ & $\begin{array}{c}\text { AmB } \\
\text { Complejo lipídico }\end{array}$ \\
\hline Nombre comercial & Fungizone ${ }^{\circledR}$ & AmBisome $®$ & Fungisome ${ }^{\circledR}$ & Amphocil/Amphotec $®$ & Abelcet $\AA$ \\
\hline Estructura & Dispersión coloidal & Liposomas esféricos & Liposomas esféricos & Discos lipídicos & Espirales lipídicos \\
\hline Dosis (mg/kg) & $0,6-1$ & $3-5$ & $1-3$ & 5 & 5 \\
\hline Vida media en plasma (h) & $\begin{array}{c}27-39 \\
\text { (dosis de } 1 \mathrm{mg} / \mathrm{kg} \text { ) }\end{array}$ & 8,6 & $24-48$ & $\begin{array}{c}28 \\
\text { (dosis de } 4 \text { mg/kg) }\end{array}$ & 16 \\
\hline Tamaño de la partícula (nanómetros) & 0,035 & $<0,080$ & $0,01 \pm 0,014$ & $0,11-0,14$ & $1,6-11$ \\
\hline Concentración en plasma $(\mu \mathrm{g} / \mathrm{mL})$ & 1,1 & 83 & 1,012 & 3,1 & 1,7 \\
\hline Penetración al LCR ( $\mu \mathrm{g} / \mathrm{mL})$ & 0,023 & 0,024 & ** & 0,014 & 0,022 \\
\hline Penetración al SNC ( $\mu \mathrm{g} / \mathrm{mL})$ & 0,18 & 1,99 & $\star *$ & 0,22 & 0,27 \\
\hline Concentración en hígado $(\mu \mathrm{g} / \mathrm{g}) 7$ h después & $26,9 \pm 0,8$ & $59,8 \pm 6,9$ & ** & ** & $57,9 \pm 5,3$ \\
\hline Concentración en grasa $(\mu \mathrm{g} / \mathrm{g}) 7 \mathrm{~h}$ después & $1,2 \pm 0,3$ & $8,9 \pm 1,9$ & ** & ** & $2,1 \pm 1,3$ \\
\hline Concentración en médula ósea 7 h después $(\mu \mathrm{g} / \mathrm{g}$ ) & $8 \pm 1,7$ & $39,5 \pm 4,7$ & ** & ** & $35,4 \pm 12,7$ \\
\hline Concentración en cerebro* $(\mu \mathrm{g} / \mathrm{g})$ & ** & $0,56^{a}$ & ** & ** & $1,6^{b}$ \\
\hline Concentración en pulmón* ( $\mu \mathrm{g} / \mathrm{g}$ ) & $12,9^{c}$ & $6,8^{\mathrm{a}}$ & ** & ** & $222^{b}$ \\
\hline
\end{tabular}


está formando un complejo con DSPG y el colesterol. Su mayor tamaño le permite escapar del aclaramiento inicial del retículo endoplasmático sarcoplásmico. Una vez captado por el retículo endoplasmático sarcoplásmico, las concentraciones en hígado y bazo aumentan conforme disminuyen a nivel plasmático. De esta manera, se garantiza que no haya residuos de AmB liposomal en sangre ${ }^{10}$. Es la forma que logra las mayores concentraciones séricas.

\section{Complejo lipídico de anfotericina $B$}

Se encuentra en el mercado bajo el nombre de Abelcet $^{\mathbb{R}}$ (Enzo Pharmaceuticals). Es una suspensión estéril para infusión intravenosa, de color opaco, compuesta de AmB unida a dos fosfolípidos en una relación molar de 1:1, la dimiristiril fosfatidil colina y el DMPG. Los lípidos se encuentran en una relación molar entre ellos de 7:3. La concentración de anfotericina dentro del complejo es de $33 \%$, su pH varía entre 5 y 7 . Su estructura tiene forma de espiral y tiene un diámetro de 2 a $5 \mu \mathrm{m}$ en promedio ${ }^{17}$.

A pesar de poseer mayor contenido de AmB, el tamaño más grande de las partículas condiciona que la toxicidad aguda sea menor. Al igual que la presentación como suspensión coloidal, es rápidamente aclarado del torrente sanguíneo y tomado por el tejido reticuloendotelial. Su pico en plasma no sobrepasa los $5 \mu \mathrm{g} / \mathrm{ml}$ a dosis terapéuticas, comparado con AmB liposomal que a dosis similares produce picos hasta 25 a 500 veces mayores sin producir mayor toxicidad. La dosis utilizada es de $5 \mathrm{mg} /$ $\mathrm{kg}$ en infusión con dextrosa; $20 \mathrm{~mL}$ corresponden a 100 mg de anfotericina ${ }^{17}$. Los estudios muestran menor nefrotoxicidad que AmB desoxicolato, pero mayor que AmB liposomal (ver más adelante). Esta forma de anfotericina no está disponible en Chile.

\section{Suspensión coloidal de AmB}

Se conoce bajo el nombre de Amphocil ${ }^{\circledR}$ (Cambridge Laboratories) y Amphotec ${ }^{\circledR}$ (Ben Venue Laboratories). En Chile se consigue Amphotec ${ }^{\circledR}$, producido por Alza Corporation (California). Es un polvo liofilizado, estéril, para infusión intravenosa. Está compuesto por AmB asociada a colesteril sulfato de sodio en una relación 1:1. Su presentación es de $50 \mathrm{mg}$ de anfotericina en $10 \mathrm{~mL}$ o $100 \mathrm{mg}$ de anfotericina en $20 \mathrm{~mL}$. Al ser reconstituido se forma la suspensión coloidal con partículas microscópicas en forma de disco, las cuales no interactúan con el plasma, proteínas o eritrocitos. Su unión a lipoproteínas es menor a $10 \%{ }^{16}$.

Después de la infusión, el aclaramiento por parte del retículo sarcoplásmico es rápido, permitiendo que se retenga en el hígado, por lo menos el 75\%, después de las primeras $24 \mathrm{~h}$ de su administración; las concentraciones séricas son menores comparadas con AmB desoxicolato. $\mathrm{Su}$ toxicidad aguda en ratas es comparable con la que produce la presentación como complejo lipídico ${ }^{11}$.

\section{Anfotericina B liposomal de la India}

Se encuentra en el mercado bajo el nombre de Fungisome $^{\circledR}$ (Lifecare Innovations). Se encuentra en presentaciones como viales de $10 \mathrm{mg}, 25 \mathrm{mg}$ y $50 \mathrm{mg}$. Son liposomas unilamelares pequeños, compuestos por fosfatidilcolina, dipalmitoil fosfatidiletanolamina $\mathrm{y}$ colesterol. Fue diseñada en búsqueda de la disminución de la toxicidad aguda y la nefrotoxicidad. Funciona de manera similar a AmB liposomal. Los estudios reportan que se une a lipoproteínas plasmáticas, lo que permite una eliminación bifásica en plasma y mayor duración del fármaco en éste. Su vida media es de 24 a $48 \mathrm{~h}^{12}$.

El estudio post-mercadeo, retrospectivo, desarrollado por Sanath y cols., de seguridad, tolerabilidad y eficacia del fármaco mostraron una tasa de respuesta exitosa de $91 \%$ con respuesta completa en $73,6 \%$ de los casos y respuesta parcial en $17,5 \%$ de los casos, a dosis de $1 \mathrm{mg} /$ $\mathrm{kg}$ /día; dosis que es sub-terapéutica para otras preparaciones lipídicas. En cuanto a los efectos adversos, los clasifican en leves, moderados y graves, con frecuencias de 36,10 y $1,8 \%$, respectivamente ${ }^{13}$. Sin embargo, no ilustra en valores los cambios en la creatininemia, no es de asignación aleatoria y tiene una pobre estandarización, limitantes importantes para su validez externa; se requieren más estudios que evalúen la efectividad de esta AmB y su toxicidad.

Las diferencias de las presentaciones de anfotericinas están consignadas en la Tabla $2^{10,12,14,17}$. En general, las concentraciones séricas y tisulares de AmB liposomal son mayores. En el hígado, médula ósea y pulmón se observan altas concentraciones del complejo lipídico.

\section{Efectos adversos}

\section{Toxicidad aguda}

La toxicidad aguda está relacionada principalmente a la infusión y se da como resultado de la producción de citoquinas pro-inflamatorias. Se presenta por lo menos en la mitad de los pacientes. Los síntomas principales son náuseas, vómitos, escalofríos, fiebre, alteraciones en la presión arterial e hipoxia. Otro efecto adverso importante es la hipercalemia secundaria a pérdida citoplasmática de potasio, lo que aumenta el riesgo de arritmias cardíacas. La hipercalemia se produce en relación a la lesión de la membrana. Esta lesión, medida en ensayos de hemólisis in vitro, es más frecuente con la formulación de dispersión coloidal, seguida de aquella en complejo lipídico. Se requieren concentraciones 1.000 veces mayores de AmB liposomal para producir el mismo nivel de daño celular ${ }^{18}$. También puede producir cardiotoxicidad con dosis superiores al rango terapéutico, que se manifiesta como arritmias ventriculares y bradicardia, lo cual se ha observado incluso con AmB liposomal ${ }^{19,20}$. Un estudio en 
infusión continua de al menos una hora en pacientes con función renal mayor a $25 \mathrm{ml}$ por min, muestra que la frecuencia de este efecto adverso es muy baja ${ }^{21}$. En pacientes con alteraciones cardíacas previas y niños, la hipercalemia puede presentarse incluso a dosis terapéuticas. La AmB administrada de forma endovenosa también tiene potencial neurotóxico y se asocia con confusión, incoherencia, delirium, obnubilación, comportamientos psicóticos y convulsiones, además de otros menos frecuentes ${ }^{8}$.

\section{Toxicidad crónica}

$\mathrm{Al}$ inicio del manejo con $\mathrm{AmB}$, por efecto de vasoconstricción de la arteriola aferente, se incrementa la creatininemia, incluso hasta el doble, en 40 a $60 \%$ de los pacientes, y en $80 \%$ aumenta por debajo de este valor. Esta vasoconstricción está mediada por el mecanismo de retroalimentación del túbulo distal secundario a un incremento en la liberación de solutos. Además, se cree que produce daño directo a las arteriolas aferentes, tejido renal y túbulo distal. Las manifestaciones clínicas de nefrotoxicidad incluyen pérdida de potasio y magnesio urinario, hipocalemia, hipomagnesemia, acidemia metabólica, diabetes insípida e insuficiencia renal ${ }^{8}$. La nefrotoxicidad de AmB se observa con mayor frecuencia en pacientes que tienen otros factores de riesgo para ella ${ }^{22}$ (Tabla 3). Un estudio en Chile, en un hospital universitario, mostró que una cuarta parte de los pacientes tenía fiebre y cerca de $10 \%$ tenían toxicidad renal a pesar de una alta frecuencia de infusión continua ${ }^{23}$. Un estudio retrospectivo de la experiencia en la vida real en Curitiba, en Brasil, mostró una altísima tasa de falla renal (superior

\section{Tabla 3. Factores de riesgo de nefrotoxicidad}

Sexo masculino

Dosis diaria $\geq 35 \mathrm{mg} /$ día

Uso de algunos medicamentos: AINES, diuréticos, ciclosporina, medios de contraste

Peso mayor a $90 \mathrm{~kg}$

Uso concomitante de antimicrobianos nefrotóxicos (aminoglucósidos, vancomicina)

Trauma o cirugía mayor

Falla renal de base

Modificado a partir de referencia 22.

a $50 \%$ ), sin identificación de otros factores de riesgo ${ }^{24}$. Es importante mencionar que este estudio utilizó criterios más estandarizados de falla renal aguda (conocidos como Acute Kidney Injury Network o AKIN, por sus siglas), más sensibles que otros criterios previamente utilizados.

La Tabla 4 muestra la frecuencia de nefrotoxicidad en estudios clínicos que han utilizado alguna forma de AmB junto con las definiciones utilizadas ${ }^{25-33}$. Esto es especialmente importante, ya que dichas definiciones han cambiado en el tiempo y la mayoría de estos artículos no utiliza la definición moderna de falla renal ${ }^{22}$.

Anfotericina B liposomal produce menor frecuencia de nefrotoxicidad. Esta diferencia se debe a la formación de un complejo molecular estabilizado por la interacción del grupo amino de AmB y el grupo fosfato del DSPG. Este complejo está respaldado por la interacción de los residuos esteres del DSPG y la porción del polieno del

\begin{tabular}{|c|c|c|c|c|}
\hline Producto & Comparador & Tasa de nefrotoxicidad & Definición de toxicidad & Referencia \\
\hline AmB desoxicolato & Fluconazol & 43 vs $19 \%$ & Aumento 1,5 veces la creatininemia basal & $(25)$ \\
\hline AmB desoxicolato & Caspofungina & 24,8 vs $8,4 \%$ & $\begin{array}{l}\text { Aumento de } 2 \text { veces la creatininemia o aumento de } 1 \mathrm{mg} / \mathrm{dl} \\
\text { en pacientes por encima de límite normal }\end{array}$ & (26) \\
\hline Fluconazol + AmB desoxicolato & Fluconazol + placebo & 23 vs $3 \%$ & & $(29)$ \\
\hline AmB liposomal & Micafungina & 3,7 vs $2,1 \%$ & $\begin{array}{l}\text { Aumento de dos veces la creatininemia o al menos un } \\
\text { incremento de } 88,4 \mu \mathrm{mol} / \mathrm{L} \text { comparado con el valor } \\
\text { de base de creatininemia }\end{array}$ & $(27)$ \\
\hline AmB liposomal & AmB complejo lipídico & 25,9 vs $62,8 \%$ & Aumento de 1,5 veces el valor de base de creatininemia & $(30)$ \\
\hline AmB liposomal & AmB complejo lipídico & 14,8 vs $42,3 \%$ & Aumento de dos veces el valor de base de creatininemia & $(30)$ \\
\hline AmB liposomal & AmB complejo lipídico & 6,2 vs $26,9 \%$ & Aumento de tres veces el valor de base de creatininemia & $(30)$ \\
\hline AmB desoxicolato & AmB complejo lipídico & 32 vs $8 \%$ & $\begin{array}{l}\text { Aumento de dos veces la creatininemia de base o un incre- } \\
\text { mento de más de } 1,5 \mathrm{mg} / \mathrm{dL}\end{array}$ & (31) \\
\hline AmB liposomal & AmB complejo lipídico & 7,1 vs $23,3 \%$ & Aumento de 1,5 veces la creatininemia de base & $(32)$ \\
\hline AmB desoxicolato & AmB liposomal & 32 vs $16 \%$ & Incremento mayor o igual al $100 \%$ de la creatininemia de base & (33) \\
\hline AmB desoxicolato & AmB liposomal & 33,7 vs $18,7 \%$ & Aumento de dos veces la creatininemia de base & $(28)$ \\
\hline
\end{tabular}


medicamento ${ }^{14}$. Todo lo anterior asegura que la disociación del complejo molecular sea menor a $1 \%$ al estar en contacto con el eritrocito, condicionando que el tamaño de AmB liposomal sea demasiado grande como para atravesar la barrera glomerular y que su aclaramiento renal en humanos sea 14 veces menor. Sólo la AmB liposomal disociada es capaz de eliminarse por vía renal.

\section{Otras toxicidades}

Anfotericina B produce supresión directa de la eritropoyesis con consecuente anemia y, en algunos casos, trombocitopenia. También se han reportado casos de hiperbilirrubinemia sin un patrón definido, lo que conlleva a la monitorización de valores de bilirrubinemia y función hepática ${ }^{8}$.

\section{Escenarios de uso de las presentaciones lipídicas de anfotericina}

\section{Candidemia}

Según documentó Kuse y cols., la eficacia de AmB liposomal en candidemia fue de $69,6 \%$ con una persistencia de $9 \%$ al completar tratamiento y con una mortalidad de $17 \%$ durante el tratamiento y de $40 \%$ a doce semanas de seguimiento ${ }^{27}$. Este fue un estudio con asignación aleatoria, controlado, comparando AmB liposomal y micafungina, con un análisis por protocolo. No hubo diferencias significativas en eficacia y mortalidad con el comparador. Los parámetros anteriores demuestran por qué el uso de la AmB liposomal es una buena alternativa de tratamiento; sin embargo, la frecuencia de efectos adversos y los costos asociados con el uso de AmB liposomal, lo ubicarían como una tercera alternativa detrás de las equinocandinas y los azoles.

\section{Neutropenia febril}

También se ha utilizado AmB liposomal en el escenario de neutropenia febril, donde los microorganismos más frecuentemente identificados son Candida y Aspergillus. En este escenario, un estudio clínico con asignación aleatoria, comparó AmB liposomal con $\mathrm{AmB}$ desoxicolato con tasas de éxito de 50,1 y 49,4\%, respectivamente ${ }^{28}$. $\mathrm{Si}$ bien la respuesta es similar, se debe resaltar la diferencia entre ambas presentaciones como causantes de nefrotoxicidad, con una diferencia de $20 \%$ a favor de la presentación liposomal, cuando se considera falla renal una elevación de la creatininemia superior a 1,5 mg/dL.

\section{Aspergilosis invasora}

El tratamiento de aspergilosis invasora consta de una fase de inducción donde la administración es por vía endovenosa, y de una fase de consolidación, por vía oral. El medicamento de elección para ambas fases es vorico- nazol $^{34,35}$. Voriconazol ha mostrado una eficacia superior a AmB con tasas de respuesta al final del tratamiento de 71 y $58 \%$, respectivamente. Los pacientes tratados con AmB experimentan, adicionalmente, tasas de toxicidad aguda y nefrotoxicidad superiores a voriconazol ${ }^{36}$.

Con el desarrollo de las formas lipídicas de AmB que han mostrado disminución en la toxicidad que su predecesora presenta, también se ha estudiado la respuesta de pacientes con aspergilosis invasora a AmB liposomal. Inicialmente se comparó $\mathrm{AmB}$ desoxicolato y $\mathrm{AmB}$ liposomal como tratamiento de primera línea en pacientes con sospecha o confirmación de aspergilosis pulmonar, ambas a las dosis terapéuticas descritas. Las tasas de respuesta fueron de 29 y $52 \%$, respectivamente ${ }^{37}$. También se ha estudiado la respuesta de pacientes con aspergilosis invasora pulmonar a AmB liposomal en diferentes dosis. En este estudio, los pacientes a los cuales se les administró AmB liposomal en dosis de $1 \mathrm{mg} / \mathrm{kg}$ /día respondieron en $64 \%$, mientras que los pacientes que recibieron $4 \mathrm{mg} / \mathrm{kg} /$ día respondieron al tratamiento en $48 \%{ }^{38}$. Esto concuerda con los hallazgos en el ensayo clínico con asignación aleatoria AmBiload donde se comparó la respuesta de pacientes con aspergilosis invasora a AmB liposomal en dosis de $10 \mathrm{mg} / \mathrm{kg} /$ día y dosis estándar. Los pacientes respondieron en mayor porcentaje a la dosis usual (50\%) frente a los que recibieron dosis altas (46\%). La tasa de supervivencia al final de estudio también fue superior para el grupo de dosis estándar frente al grupo de dosis altas (93 y $88 \%$, respectivamente). Teniendo en cuenta que los pacientes que recibieron dosis de $10 \mathrm{mg} / \mathrm{kg} /$ día presentaron más toxicidad aguda y nefrotoxicidad, la recomendación es el uso de dosis estándar ${ }^{39}$.

Un estudio con asignación aleatoria comparó la eficacia de AmB desoxicolato y la suspensión coloidal en pacientes inmunocomprometidos, con aspergilosis invasora $^{40}$. Las tasas de eficacia clínica fueron similares (51 vs $52 \%$ ), al igual que la mortalidad, pero la toxicidad renal fue de $25 \%$ con la suspensión coloidal y de $49 \%$ con AmB desoxicolato.

El uso de AmB liposomal en pacientes con aspergilosis invasora se podría considerar en pacientes con interacciones importantes entre los medicamentos, pacientes que no responden a voriconazol o que son intolerantes a azoles y aquellos con falla hepática grave.

Por otro lado, la combinación de AmB con otros antifúngicos parece ser otra alternativa para el manejo de aspergilosis invasora. En el ensayo clínico con asignación aleatoria Combistrat, se comparó AmB liposomal en altas dosis con la combinación de caspofungina más $\mathrm{AmB}$ liposomal en dosis estándar. La respuesta a la terapia combinada fue superior que la respuesta a la monoterapia (67 y $27 \%$, respectivamente) ${ }^{41}$. Sin embargo, se requiere de mayor exploración en terapias combinada con AmB liposomal. 


\section{Histoplasmosis}

Clásicamente, el tratamiento de primera línea para histoplasmosis diseminada ha sido los polienos e itraconazol en algunos casos ${ }^{42}$.

Sin embargo, AmB liposomal ha demostrado su efectividad como manejo de primera línea, especialmente en pacientes con infección por VIH/SIDA. Un estudio que incluyó información clínica y microbiológica de dos estudios con asignación aleatoria comparó itraconazol y AmB liposomal (dosis de $3 \mathrm{mg} / \mathrm{kg} / \mathrm{día}$ ) en pacientes con SIDA cursando con un primer episodio de histoplasmosis adquirida; en él se observó respuestas de 85 y $86 \%$, respectivamente. A pesar de respuestas similares, AmB liposomal logró un mayor aclaramiento del hongo con concentraciones de antígeno menores y una negativización en los hemocultivos más rápida: a las dos semanas en $85 \%$ de los pacientes frente a sólo $53 \%$ de los pacientes con itraconazol ${ }^{43}$.

También se ha comparado la eficacia de AmB liposomal frente a AmB desoxicolato como terapia de primera línea en pacientes seopositivos para VIH en un estudio con asignación aleatoria ${ }^{44}$. En la fase de dos semanas de inducción, el grupo tratado con AmB liposomal mostró una mayor respuesta frente a $\mathrm{AmB}$ desoxicolato, con tasas de 88 y $64 \%$, respectivamente. Adicionalmente, el grupo tratado con AmB liposomal mostró menores tasas de mortalidad, efectos adversos secundarios a la infusión y nefrotoxicidad. Sin embargo, este es un estudio pequeño y no se ha reproducido estos hallazgos por otros investigadores. Las respuestas y efectos adversos en general, fueron similares en las diez semanas siguientes de consolidación ${ }^{44}$. Esta información sugiere que AmB liposomal sería de primera elección en pacientes con infección por VIH y una alternativa en pacientes sin esta infección.

\section{Zigomicosis}

El tratamiento de elección para zigomicosis ha sido AmB desoxicolato y con el advenimiento de las formas lipídicas, éstas han tomado el protagonismo. Hay múltiples estudios comparando ambas presentaciones de AmB.

En pacientes con malignidades hematológicas, a dosis estándares, AmB liposomal mostró una respuesta superior (58\%) frente a $\mathrm{AmB}$ desoxicolato que presentó una respuesta de $23 \%{ }^{45}$. Vale la pena mencionar que éste fue un estudio retrospectivo, sin asignación aleatoria, donde el beneficio del uso de AmB liposomal se mantuvo al utilizar modelos multivariados para ajustar los resultados.

Además de la respuesta a AmB liposomal, también se ha estudiado la respuesta de pacientes con zigomicosis a otras formas lipídicas de AmB. En una serie de casos, se evaluó la eficacia de AmB de dispersión coloidal como segunda línea de tratamiento en este grupo. Las tasas de respuesta fueron de $60 \%{ }^{46}$.
A pesar de que se ha investigado el posaconazol como posible alternativa de primera línea, no ha sido posible compararlo con AmBs porque su presentación oral lo limita (actualmente se realizan estudios con presentaciones endovenosas). Sin embargo, cabe destacar que a pesar de su alto potencial de interacciones medicamentosas, ha mostrado una eficacia comparable con AmB desoxicolato y AmB liposomal. Series de casos en las que se ha usado como segunda línea en pacientes que no han tolerado o no han respondido a otros antifúngicos, reportan tasas de respuesta de 60 a $79 \% \%^{47,48}$.

Por lo pronto, las formas lipídicas de AmB continúan como esquema de primera línea de zigomicosis en pacientes con alto riesgo de nefrotoxicidad; sin embargo, sobre su beneficio en pacientes sin este factor de riesgo no hay evidencia que permita definirlo. Aún queda la pregunta de si la eficacia de AmB liposomal aumenta si se administran dosis mayores sin aumentar los efectos tóxicos. El estudio prospectivo Ambizygo se encuentra en desarrollo en búsqueda de una respuesta a este interrogante.

\section{Criptococosis}

Anfotericina B, en combinación con flucitosina, es el medicamento de elección para el tratamiento de la criptococosis ${ }^{49,50}$. La resistencia a antimicóticos ha sido un problema importante. Un estudio realizado en Brasil recuperó en ocho casos Cryptococcus gatti y en 176 casos C. neoformans. De los casos considerados como intrahospitalarios, $10,5 \%$ fueron resistentes a AmB; mientras que de 81 aislados adquiridos en la comunidad, 6,2\% fueron resistentes a fluconazol. En los ocho casos causados por $C$. gatti no se encontró resistencia a $\mathrm{AmB}^{51}$. Otros estudios han demostrado el aumento de la resistencia de estos microorganismos a fluconazol, demostrando incluso resistencias de $100 \%$ a este medicamento ${ }^{52}$.

La resistencia a AmB encontrada en Brasil es concordante con el estudio realizado por Pfaller y cols., quienes encontraron una resistencia de $8 \%$ a AmB desoxicolato ${ }^{53}$. En general, las tasas de resistencia a $\mathrm{AmB}$ han sido bajas, incluso en pacientes adultos inmunocomprometidos, en los que se demostró una resistencia $<1 \% \%^{54,55}$. En Colombia, según la encuesta nacional de criptococosis, la mayoría de los casos son presentados en pacientes con infección por VIH/SIDA $(83,5 \%)$ de los cuales se desarrollan en hombres $76,6 \%$. La especie prevalente es $C$. neoformans ( $96,6 \%)$, con resistencia de $18 \%$ a fluconazol. El 30\% presentó una respuesta dosis dependiente ${ }^{56}$. De acuerdo al estudio de vigilancia Artemis, un escenario similar se ha observado en América Latina donde las tasas de resistencia encontradas para C. neoformans frente a fluconazol en la región fueron de 13,6\% en el período entre 1997 y $2007^{57}$.

Es clara la prevalencia de esta infección entre pacientes 
inmunocomprometidos como también lo es el aumento de la resistencia de estos microorganismos a fluconazol, lo cual se puede explicar por su uso continuo como profilaxis. Con base en lo anterior, se ha considerado que AmB desoxicolato debe ser la primera línea de tratamiento debido a su efectividad y baja resistencia; sin embargo, la toxicidad aguda y crónica asociada a ésta ha llevado a contemplar las formas lipídicas como una mejor opción de manejo.

Un estudio no aleatorio en pacientes receptores de trasplantes de órganos sólidos, con criptococosis en el sistema nervioso central, mostró una disminución en la mortalidad al emplear formas lipídicas de anfotericina comparados con los pacientes tratados con $\mathrm{AmB}$ desoxicolato con tasas de mortalidad de 10,9 y $40 \%$, respectivamente ${ }^{58}$.

Un estudio aleatorio, pequeño, comparó AmB liposomal con AmB desoxicolato. El estudio mostró una mayor erradicación microbiológica mediante el uso de AmB liposomal en la inducción ${ }^{59}$.

Las principales ventajas de la forma liposomal sobre las demás formas lipídicas son la regulación negativa en la expresión genética de citoquinas pro-inflamatorias, lo que lleva a un menor daño tisular; la mejor distribución del medicamento con menor afinidad por celular mamíferas y la baja nefrotoxicidad ${ }^{60}$. Todo lo anterior, lleva a la conclusión de que AmB liposomal puede ser una opción para el tratamiento de la criptococosis y podría ser manejada como primera línea en pacientes con alto riesgo de nefrotoxicidad.

\section{Leishmaniasis}

La incidencia de leishmaniasis ha sido altamente estudiada y es de gran importancia en América Latina. La incidencia mundial de leishmaniasis visceral es de 58.227 casos de los cuales 3.668 casos $(6,2 \%)$ se presentan en América, la segunda incidencia más alta en el mundo. Dentro de esta región, el primer lugar en incidencia lo ocupa Brasil con 3.481 casos seguido por Colombia. Por su parte, la leishmaniasis cutánea representa 214.036 casos mundiales, de los cuales la región de América aportó 66.941 casos $(31,3 \%)$. Dentro de esta región, el primer lugar lo ocupa nuevamente Brasil con 26.008 casos seguido por Colombia con 17.420 casos $^{61}$.

La primera línea de tratamiento es bien conocida y la representa los antimonios pentavalentes. En los últimos años, se ha notado un aumento en la resistencia y; una baja adherencia y tolerancia por la alta toxicidad y comorbilidad a este medicamento. Por esto se han buscado alternativas terapéuticas.

El parásito requiere de la interacción con el colesterol de la membrana del macrófago para ingresar a éste. Al crear depleción e, incluso, secuestro del colesterol, se evita un número mayor de amastigotes intracelulares ${ }^{62}$. Con base en esto y en el conocimiento de la estructura del parásito con alto contenido de ergosterol, se propuso a AmB como segunda línea de manejo y, dentro de éstas, las formas lipídicas, teniendo en cuenta su capacidad para producir menor nefrotoxicidad ${ }^{63}$, y la alta tasa de respuesta que se encuentra alrededor de $97 \%$ de los casos, sin haberse reportado resistencia ${ }^{64}$.

Dentro de las formas lipídicas se prefiere AmB liposomal, ya que muestra una mayor efectividad, un aumento en su biodisponibilidad y disminución clara de los efectos adversos ${ }^{65}$. El uso de AmB liposomal estaría reservado a los pacientes con leishmaniasis visceral, diseminada y muco-cutánea que ha fallado al tratamiento previo con antimoniales. Existe limitada información con las diferentes formas de $\mathrm{AmB}^{66}$, aunque un estudio español mostró que en pacientes con infección por VIH, AmB en complejo lipídico disminuye la frecuencia de recaída al ser administrada como profilaxis secundaria ${ }^{67}$.

\section{Conclusiones}

Anfotericina B es un antimicótico de primera línea para el manejo de las zigomicosis, histoplasmosis y criptococosis; es una alternativa para pacientes con fallo terapéutico e infecciones por Candida spp. o Aspergillus spp. Anfotericina en presentación liposomal pueden contribuir a una menor toxicidad, especialmente sobre el riñón. Aunque en países como E.U.A., el uso de AmB desoxicolato ha quedado prácticamente proscrito por su perfil de efectos adversos, es un medicamento muy económico (probablemente menos de $\$ 50$ dólares por día) y de mayor uso que otros antimicóticos ${ }^{2}$. Entonces, ¿cuál podría ser el escenario de uso en la práctica en nuestra región? Probablemente sea posible iniciar AmB desoxicolato en los pacientes con indicación para su uso, maximizando las estrategias de prevención de la toxicidad aguda y la nefrotoxicidad. En aquellos en los que, a pesar de estas estrategias se presente la falla renal, o en aquellos con varios factores de riesgo (más de dos) o en los cuales se desea preservar la función renal (por ej. los pacientes receptores de trasplante renal o de otros órganos sólidos), podría utilizarse anfotericinas en presentaciones lipídicas, de acuerdo con la indicación y la disponibilidad en cada país ${ }^{22}$. Vale la pena mencionar que hay información limitada de efectividad para las formas de complejo lipídico y dispersión coloidal, así como para la anfotericina liposomal procedente de la India. Es posible, que cada país tenga que hacer una evaluación de costoefectividad local para determinar en qué ocasiones puede ser costo-efectiva o se tenga la disponibilidad económica para hacer dicho pago.

Agradecimiento. A Anita Montañez por su apoyo administrativo. 


\section{Resumen}

Dado el aumento en el número de pacientes inmunosuprimidos en los últimos años, el uso de anfotericina B desoxicolato también se incrementó debido a una mayor incidencia de las infecciones fúngicas invasoras en esta población. Este medicamento tiene una alta frecuencia de efectos adversos, especialmente nefrotoxicidad. Se han desarrollado modificaciones de la presentación de anfotericina B con el desarrollo de formas lipídicas (liposomal, complejo lipídico, suspensión coloidal y fórmula liposomal procedente de la India) que tienen el mismo espectro y con variaciones en su efectividad y toxicidad. Se presenta una revisión de las formas lipídicas de anfotericina, sus diferencias en efectividad $\mathrm{y}$, especialmente, nefrotoxicidad. El principal problema para su implementación en América Latina es el alto costo de estas presentaciones.

\section{Referencias bibliográficas}

1.- Klepser M. The value of amphotericin B in the treatment of invasive fungal infections. J Crit Care 2011; 26: 225 e1-10.

2.- Nucci M, Queiroz-Telles F, Alvarado-Matute T, Tiraboschi I N, Cortes J, Zurita J, et al. Epidemiology of candidemia in Latin America: a laboratory-based survey. PLoS One 2013; 8: e59373.

3.- Bergamasco M D, Garnica M, Colombo A L, Nucci M. Epidemiology of candidemia in patients with hematologic malignancies and solid tumours in Brazil. Mycoses 2013; 56: 256-63.

4.- Ajenjo H M, Aquevedo S A, Guzmán D A, Poggi M H, Calvo A M, Castillo V C, et al. Epidemiologial profile of invasive candidiasis in intensive care units at a university hospital. Rev Chilena Infectol 2011; 28: 118-22.

5.- Martínez R. An update on the use of antifungal agents. J Bras Pneumol 2006; 32: 449-60.

6.- Schmalreck A F, Willinger B, Haase G, Blum G, Lass-Florl C, Fegeler W, et al. Species and susceptibility distribution of 1,062 clinical yeast isolates to azoles, echinocandins, flucytosine and amphotericin B from a multicentre study. Mycoses 2012; 55: e124-37.

7.- Baginski M, Czub J. Amphotericin B and its new derivatives-mode of action. Curr Drug Metab 2009; 10: 459-69.

8.- Laniado-Laborin R, Cabrales-Vargas M N. Amphotericin B: side effects and toxicity. Rev Iberoam Micol 2009; 26: 223-7.

9.- Lemke A, Kiderlen A F, Kayser O. Amphotericin B. Appl Microbiol Biotechnol 2005; 68: 151-62.

10.- Azanza Perea J R, Barberan J. Anfotericina B forma liposomal: un perfil farmacocinético exclusivo. Una historia incabada. Rev Esp Quimioter 2012; 25: 17-24.

11.- Fielding R M, Smith P C, Wang L H, Porter J, Guo L S. Comparative pharmacokinetics of amphotericin B after administration of a novel colloidal delivery system, $\mathrm{ABCD}$, and a conventional formulation to rats. Antimicrob Agents Chemother 1991; 35: 1208-13.

12.- Kshirsagar N A, Pandya S K, Kirodian G B,
Sanath S. Liposomal drug delivery system from laboratory to clinic. J Postgrad Med 2005; 51 Suppl 1: S5-15.

13.- Sanath S S, Gogtay N J, Kshirsagar N A. Post-marketing study to assess the safety, tolerability and effectiveness of Fungisome: an Indian liposomal amphotericin B preparation. J Postgrad Med 2005; 51 Suppl 1: S58-63.

14.- Adler-Moore J P, Proffitt R T. Amphotericin B lipid preparations: what are the differences? Clin Microbiol Infect 2008; 14 Suppl 4: 25-36.

15.- Hamill R J. Amphotericin B formulations: a comparative review of efficacy and toxicity. Drugs 2013; 73: 919-34.

16.- Patel R. Amphotericin B colloidal dispersion. Expert Opin Pharmacother 2000; 1: 475-88.

17.- Antoniadou A, Dupont B. Lipid formulations of amphotericin B: where are we today? J Mycol Med 2005; 14: 230-8.

18.- Jensen G M, Skenes C R, Bunch T H, Weissman C A A, Satorius N, Moynihan A, et al. Determination of the relative toxicity of amphotericin B formulations: a red blood cell potassium release assay. Drug Deliv 1999; 6.

19.- Aguado J M, Hidalgo M, Moya I, Alcazar J M, Jiménez M J, Noriega A R. Ventricular arrhythmias with conventional and liposomal amphotericin. Lancet 1993; 342: 1239.

20.- Groot O A, Trof R J, Girbes A R, Swart N L, Beishuizen A. Acute refractory hyperkalaemia and fatal cardiac arrest related to administration of liposomal amphotericin B. Neth J Med 2008; 66: 433-7.

21.- Bowler W A, Weiss P J, Hill H E, Hoffmeister K A, Fleck R P, Blacky A R, et al. Risk of ventricular dysrhythmias during 1-hour infusions of amphotericin B in patients with preserved renal function. Antimicrob Agents Chemother 1992; 36: 2542-3.

22.- Cortés J A, Soto R, Álvarez C A, Buitrago G, Camargo R, Cataño J C, et al. Consenso de uso de antimicrobianos en pacientes críticamente enfermos con falla renal o en riesgo de padecerla. Infectio 2011; 15: 49-63.

23.- Quinteros A R, Fica C A, Abusada A N, Muñoz C L, Novoa M C, Gallardo A C. Uso de anfotericina $\mathrm{B}$ deoxicolato y sus reacciones adversas en un hospital universitario en Chile. Rev Chilena Infectol 2010; 27: 25-33.

24.- Tuon F F, Koenig F, Jacometto D, Rocha J L. Are there risk factors for acute renal failure in adult patients using deoxycholate amphotericin B? Rev Iberoam Micol 2013; 30: 21-4.

25.- Phillips P, Shafran S, Garber G, Rotstein C, Smaill F, Fong I, et al. Multicenter randomized trial of fluconazole versus amphotericin B for treatment of candidemia in non-neutropenic patients. Canadian Candidemia Study Group. Eur J Clin Microbiol Infect Dis 1997; 16: $337-45$.

26.- Mora-Duarte J, Betts R, Rotstein C, Colombo A L, Thompson-Moya L, Smietana J, et al. Comparison of caspofungin and amphotericin B for invasive candidiasis. N Engl J Med 2002; 347: 2020-9.

27.- Kuse E R, Chetchotisakd P, da Cunha C A, Ruhnke M, Barrios C, Raghunadharao D, et al. Micafungin versus liposomal amphotericin B for candidaemia and invasive candidosis: a phase III randomised double-blind trial. Lancet 2007; 369: 1519-27.

28.- Walsh T J, Finberg R W, Arndt C, Hiemenz J, Schwartz C, Bodensteiner D, et al. Liposomal amphotericin B for empirical therapy in patients with persistent fever and neutropenia. National Institute of Allergy and Infectious Diseases Mycoses Study Group. N Engl J Med 1999; 340: 764-71.

29.- Rex J H, Pappas P G, Karchmer A W, Sobel J, Edwards J E, Hadley S, et al. A randomized and blinded multicenter trial of high-dose fluconazole plus placebo versus fluconazole plus amphotericin B as therapy for candidemia and its consequences in nonneutropenic subjects. Clin Infect Dis 2003; 36: 1221-8.

30.- Wingard J R, White M H, Anaissie E, Raffalli J, Goodman J, Arrieta A. A randomized, doubleblind comparative trial evaluating the safety of liposomal amphotericin B versus amphotericin $\mathrm{B}$ lipid complex in the empirical treatment of febrile neutropenia. L Amph/ABLC Collaborative Study Group. Clin Infect Dis 2000; 31: 1155-63.

31.- Subira M, Martino R, Gómez L, Marti J M, Estany C, Sierra J. Low-dose amphotericin B 
lipid complex vs conventional amphotericin $B$ for empirical antifungal therapy of neutropenic fever in patients with hematologic malignancies-a randomized, controlled trial. Eur J Haematol 2004; 72: 342-7.

32.- Saliba F, Cheron N, Ichai P, Renault A, Sirvent A, Depil S, et al. 123 A prospective French National Survey to assess renal safety of amphotericin B lipid complex and liposomal amphotericin B. Int J Infect Dis 2006; 10: s68-s69.

33.- Prentice H G, Hann I M, Herbrecht R, Aoun M, Kvaloy S, Catovsky D, et al. A randomized comparison of liposomal versus conventional amphotericin B for the treatment of pyrexia of unknown origin in neutropenic patients. $\mathrm{Br} \mathrm{J}$ Haematol 1997; 98: 711-8.

34.- Lanternier F, Lortholary O. Liposomal amphotericin B: what is its role in 2008 ? Clin Microbiol Infect 2008; 14 Suppl 4: 71-83.

35.- Walsh T J, Anaissie E J, Denning D W, Herbrecht R, Kontoyiannis D P, Marr K A, et al. Treatment of aspergillosis: clinical practice guidelines of the Infectious Diseases Society of America. Clin Infect Dis 2008; 46: 327-60.

36.- Herbrecht R, Denning D W, Patterson T F, Bennett J E, Greene R E, Oestmann J W, et al. Voriconazole versus amphotericin $\mathrm{B}$ for primary therapy of invasive aspergillosis. N Engl J Med 2002; 347: 408-15.

37.- Leenders A C, Daenen S, Jansen R L, Hop W C, Lowenberg B, Wijermans P W, et al. Liposomal amphotericin B compared with amphotericin B deoxycholate in the treatment of documented and suspected neutropenia-associated invasive fungal infections. Br J Haematol 1998; 103: 205-12.

38.- Ellis M, Spence D, de Pauw B, Meunier F, Marinus A, Collette L, et al. An EORTC international multicenter randomized trial (EORTC number 19923) comparing two dosages of liposomal amphotericin B for treatment of invasive aspergillosis. Clin Infect Dis 1998; 27: 1406-12.

39.- Cornely O A, Maertens J, Bresnik M, Ebrahimi R, Ullmann A J, Bouza E, et al. Liposomal amphotericin B as initial therapy for invasive mold infection: a randomized trial comparing a high-loading dose regimen with standard dosing (AmBiLoad trial). Clin Infect Dis 2007; 44: 1289-97.

40.- Bowden R, Chandrasekar P, White M H, Li X, Pietrelli L, Gurwith M, et al. A double-blind, randomized, controlled trial of amphotericin B colloidal dispersion versus amphotericin B for treatment of invasive aspergillosis in immunocompromised patients. Clin Infect Dis 2002; 35: 359-66.

41.- Caillot D, Thiebaut A, Herbrecht R, de Botton S, Pigneux A, Bernard F, et al. Liposomal amphotericin $\mathrm{B}$ in combination with caspofungin for invasive aspergillosis in patients with hematologic malignancies: a randomized pilot study (Combistrat trial). Cancer 2007; 110: 2740-6.

42.- Wheat J, Sarosi G, McKinsey D, Hamill R, Bradsher R, Johnson P, et al. Practice guidelines for the management of patients with histoplasmosis. Infectious Diseases Society of America. Clin Infect Dis 2000; 30: 688-95.

43.- Wheat L J, Cloud G, Johnson P C, Connolly P, Goldman M, Le Monte A, et al. Clearance of fungal burden during treatment of disseminated histoplasmosis with liposomal amphotericin $B$ versus itraconazole. Antimicrob Agents Chemother 2001; 45: 2354-7.

44.- Johnson P C, Wheat L J, Cloud G A, Goldman M, Lancaster D, Bamberger D M, et al. Safety and efficacy of liposomal amphotericin B compared with conventional amphotericin B for induction therapy of histoplasmosis in patients with AIDS. Ann Intern Med 2002; 137: 105-9.

45.- Pagano L, Offidani M, Fianchi L, Nosari A, Candoni A, Piccardi M, et al. Mucormycosis in hematologic patients. Haematologica 2004; 89: 207-14.

46.- Herbrecht R, Letscher-Bru V, Bowden R A Kusne S, Anaissie E J, Graybill J R, et al. Treatment of 21 cases of invasive mucormycosis with amphotericin B colloidal dispersion. Eur J Clin Microbiol Infect Dis 2001; 20: 460-6.

47.- Greenberg R N, Mullane K, van Burik J A, Raad I, Abzug M J, Anstead G, et al. Posaconazole as salvage therapy for zygomycosis. Antimicrob Agents Chemother 2006; 50: 126-33.

48.- van Burik J A, Hare R S, Solomon H F, Corrado M L, Kontoyiannis D P. Posaconazole is effective as salvage therapy in zygomycosis: a retrospective summary of 91 cases. Clin Infect Dis 2006; 42: e61-5.

49.- Perfect J R, Dismukes W E, Dromer F, Goldman D L, Graybill J R, Hamill R J, et al. Clinical practice guidelines for the management of cryptococcal disease: 2010 update by the Infectious Diseases Society of America. Clin Infect Dis 2010; 50: 291-322.

50.- Day J N, Chau T T, Wolbers M, Mai P P, Dung N T, Mai N H, et al. Combination antifungal therapy for cryptococcal meningitis. N Engl J Med 2013; 368: 1291-302.

51.- Andrade-Silva L, Ferreira-Paim K, Mora D J, Silva P R, Andrade A A, Araujo N E, et al. Susceptibility profile of clinical and environmental isolates of Cryptococcus neoformans and Cryptococcus gattii in Uberaba, Minas Gerais, Brazil. Med Mycol 2013.

52.- García-Martos P, Noval J F, García-Tapia A, Marin P, Puerto J L, Sepúlveda A. Susceptibility to antifungal agents of Cryptococcus species of clinical interest. Med Clin (Barc) 2002; 119 : 211-3.
53.- Pfaller M A, Messer S A, Boyken L, Rice C, Tendolkar S, Hollis R J, et al. Global trends in the antifungal susceptibility of Cryptococcus neoformans (1990 to 2004). J Clin Microbiol 2005; 43: 2163-7.

54.- Souza L K, Fernandes Ode F, Kobayashi C C, Passos X S, Costa C R, Lemos J A, et al. Antifungal susceptibilities of clinical and environmental isolates of Cryptococcus neoformans in Goiania city, Goias, Brazil. Rev Inst Med Trop Sao Paulo 2005; 47: 253-6.

55.- López-Jodra O, Torres-Rodríguez J M, Méndez-Vásquez R, Ribas-Forcadell E, Morera-López Y, Baro-Tomas T, et al. In vitro susceptibility of Cryptococcus neoformans isolates to five antifungal drugs using a colorimetric system and the reference microbroth method. J Antimicrob Chemother 2000; 45: 645-9.

56.- Escandon P, de Bedout C, Lizarazo J, Agudelo C I, Tobon A, Bello S, et al. Cryptococcosis in Colombia: Results of the national surveillance program for the years 2006-2010. Biomedica 2012; 32: 386-98.

57.- Pfaller M A, Diekema D J, Gibbs D L, Newell V A, Bijie H, Dzierzanowska D, et al. Results from the ARTEMIS DISK Global Antifungal Surveillance Study, 1997 to 2007: 10.5-year analysis of susceptibilities of noncandidal yeast species to fluconazole and voriconazole determined by CLSI standardized disk diffusion testing. J Clin Microbiol 2009; 47: 117-23.

58.- Sun H Y, Alexander B D, Lortholary O, Dromer F, Forrest G N, Lyon G M, et al Lipid formulations of amphotericin $\mathrm{B}$ significantly improve outcome in solid organ transplant recipients with central nervous system cryptococcosis. Clin Infect Dis 2009; 49: 1721-8.

59.- Leenders A C, Reiss P, Portegies P, Clezy K, Hop W C, Hoy J, et al. Liposomal amphotericin B (AmBisome) compared with amphotericin $\mathrm{B}$ both followed by oral fluconazole in the treatment of AIDS-associated cryptococcal meningitis. AIDS 1997; 11: 1463-71.

60.- Takemoto K, Yamamoto Y, Ueda Y. Influence of the progression of cryptococcal meningitis on brain penetration and efficacy of AmBisome in a murine model. Chemotherapy 2006; 52 : 271-8.

61.- Alvar J, Vélez I D, Bern C, Herrero M, Desjeux P, Cano J, et al. Leishmaniasis worldwide and global estimates of its incidence. PLoS One 2012; 7: e35671.

62.- Pucadyil T J, Tewary P, Madhubala R, Chattopadhyay A. Cholesterol is required for Leishmania donovani infection: implications in leishmaniasis. Mol Biochem Parasitol 2004; 133: $145-52$.

63.- Chattopadhyay A, Jafurulla M. A novel mechanism for an old drug: amphotericin B in 
the treatment of visceral leishmaniasis. Biochem Biophys Res Commun 2011; 416: 7-12.

64.- Wasan K M, Wasan E K, Gershkovich P, Zhu X, Tidwell R R, Werbovetz K A, et al. Highly effective oral amphotericin $B$ formulation against murine visceral leishmaniasis. J Infect Dis 2009; 200: 357-60

65.- Bekersky I, Fielding R M, Dressler D E, Lee J W, Buell D N, Walsh T J. Plasma protein binding of amphotericin $\mathrm{B}$ and pharmacokinetics of bound versus unbound amphotericin B after administration of intravenous liposomal amphotericin B (AmBisome) and amphotericin $\mathrm{B}$ deoxycholate. Antimicrob Agents Chemother 2002; 46 : 834-40.

66.- Laguna F, Videla S, Jiménez-Mejías M E, Sirera G, Torre-Cisneros J, Ribera E, et al. Amphotericin B lipid complex versus meglumine antimoniate in the treatment of visceral leishmaniasis in patients infected with HIV: a randomized pilot study. J Antimicrob Chemother 2003; 52: 464-8.

67.- López-Velez R, Videla S, Márquez M, Boix V, Jiménez-Mejías M E, Gorgolas M, et al. Amphotericin B lipid complex versus no treatment in the secondary prophylaxis of visceral leishmaniasis in HIV-infected patients. J Antimicrob Chemother 2004; 53: 540-3. 